\title{
Factors influencing tuberculosis screening in healthcare workers in Portugal
}

\author{
To the Editor:
}

Although the incidence of tuberculosis (TB) has increased in healthcare workers (HCWs) [1-3], several studies have shown that HCWs are not compliant with screening and/or preventive measures [4-7]. For example, a Portuguese study found that the estimated TB incidence was three- to seven-fold higher in HCWs than in the general population [8]. Latent TB infection (LTBI) diagnosis and treatment constitute the core of TB elimination, integrating the post-2015 strategies of the World Health Organization $[9,10]$.

To evaluate TB screening practices among HCWs and their reasons for nonadherence in Portugal, we developed a survey and distributed it to nurses and physicians from December 25, 2012 to January 31, 2013, closing when we received fewer than one response per day. The survey was anonymous, voluntary and digitally distributed through our network of contacts using a "snowball" distribution method where volunteers subsequently distribute the questionnaire to their contacts and so forth.

Continuous data are presented as mean $\pm \mathrm{SD}$ and compared using t-tests. Categorical data are presented as n (\%) and compared using the Chi-squared or Fisher's test, as appropriate. Multiple logistic regression analysis was used to identify statistically significant determinants of TB infection, exposure and screening. Crude and adjusted odds ratios and 95\% confidence intervals were determined. Goodness-of-fit of the models was evaluated; comparisons with the null model used the difference of deviances, as determined by Chi-squared tests, while comparisons with the saturated model used the difference of deviances, determined using the Chi-squared or Hosmer-Lemeshow test, as appropriate. The area under the receiver operating characteristic (ROC) curve was calculated for each model. All statistical analyses were performed using the R language and software, version 2.12.1 [11]. The level of significance was fixed at 0.05.

In Portugal, in 2008, there were 38932 physicians and 56859 nurses registered by their respective boards [12].

We obtained 2414 responses, of which 399 did not meet the inclusion criteria. Thus, responses from 2015 subjects were analysed; table 1 summarises the results. Of these subjects, 1540 (76.4\%) were females and $1133(56.2 \%)$ nurses. Subjects were aged 18-73 years (mean \pm sD $39.02 \pm 10.60$ years). 44 (2.2\%) subjects had a history of TB (20 before beginning professional activity).

Of the 2015 subjects, 784 (39.5\%) were never screened; of these, 741 (94.5\%) reported they were never offered screening. The remaining $43(5.5 \%)$ subjects refused screening, five $(11.6 \%)$ because they were unavailable for screening, $13(30.2 \%)$ because they saw no benefits in screening, six $(14.0 \%)$ because they would not be treated if positive and eight (18.6\%) for other reasons; $12(27.6 \%)$ did not state a reason. Among the 741 subjects who were not offered screening, 580 (78.2\%) stated that they would be screened if offered, whereas $141(19.0 \%)$ were either undecided or did not want to be screened; of the latter, 46 (32.6\%) were concerned about the side-effects of possible treatment, 18 (12.8\%) did not believe in the benefits of preventive treatment, $43(30.5 \%)$ were not sufficiently informed to make a decision and 16 (11.6\%) cited other reasons.

Of 1187 (58.9\%) subjects screened, 139 (11.7\%) were positive for LTBI (defined as the absence of disease but a positive tuberculin skin test (TST) or interferon- $\gamma$ assay); of these, 72 (51.8\%) were treated, 47 (65.2\%) after beginning professional activity. Of the 67 (48.2\%) subjects who were not treated for LTBI, 12 (17.9\%) refused treatment due to concerns about the side effects of treatment, five (7.5\%) did not believe in the benefits of treatment, nine (13.4\%) were not sufficiently informed to make a decision and 16 (23.9\%) stated other reasons. LTBI was diagnosed on routine screening in 50 subjects (36.0\%) and active post-exposure (any exposure, regardless of duration or place of exposure) in 45 (32.4\%).

Logistic regression models disregarded survey responses from 53 subjects due to the absence of at least one of the explanatory variables. Thus, 1962 subjects were included in the model estimation. All variables, except for the variable representing the youngest individuals in the exposure model, were found to have a statistically significant effect on the response. All models were shown to have a goodness of fit that was significantly better than the null model $(\mathrm{p}<0.001)$ and not significantly different from that of the saturated model ( $p=0.560$ for the screening model; $p=1.000$ for the infection and the exposure model). The area under the ROC curve was $62.1 \%$ for the screening model, $64.3 \%$ for the infection model and $59.7 \%$ for the exposure model. 
TABLE 1 Summary of results and statistical analysis

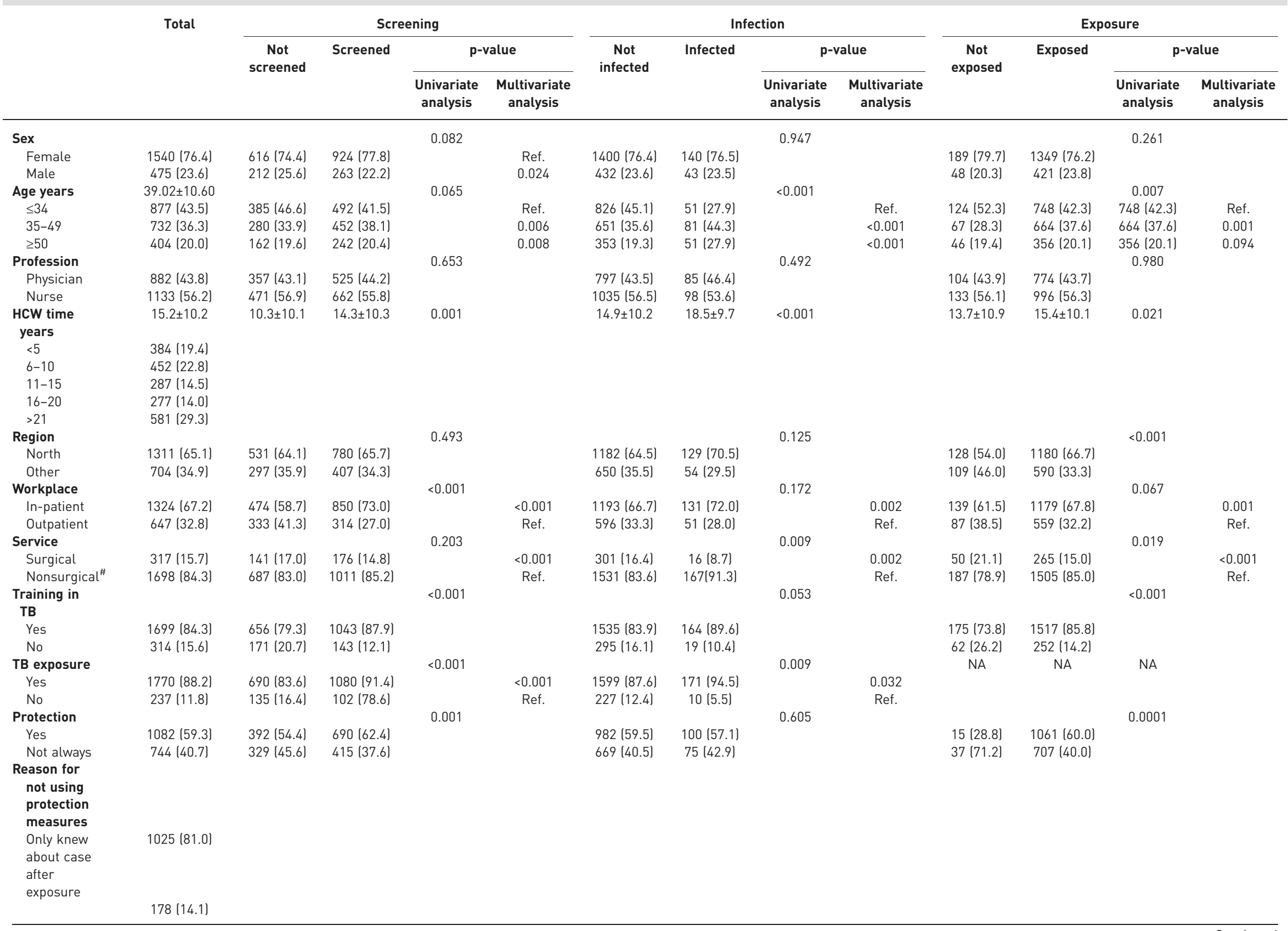


Total

\begin{tabular}{|c|c|c|c|}
\hline \multicolumn{4}{|c|}{ Screening } \\
\hline \multirow{2}{*}{$\begin{array}{c}\text { Not } \\
\text { screened }\end{array}$} & Screened & & alue \\
\hline & & $\begin{array}{c}\text { Univariate } \\
\text { analysis }\end{array}$ & $\begin{array}{l}\text { Multivariate } \\
\text { analysis }\end{array}$ \\
\hline
\end{tabular}

$15(1.2)$

$16(1.3)$

Discomfort/

carelessness

Mask not

Previous TB

Yes

No
Previous LTBI

Yes

NTB

treatment

Yes

Yes

HCW when

treated

Yes

No
HCW time

when treated

years

$\leq 5$

6-10

11-15

16-20

$\geq 21$

Yes

No
Type of

screening

Routine

Post-exposure

44 (2.2)

72 (51.8)

$33(31.4)$

37 (51.4)

$13(18.1)$

$13(18.1)$

$4(5.6)$

$5(6.9)$
$44(2.2)$
$1971(97.8)$

$139(6.9)$

1876 (93.1)

67 (48.2)

72 (68.6)

$8.14 \pm 7.69$

1187 (58.9)

$828(41.1)$

NA

NA

Data are presented as $\mathrm{n}(\%)$ or mean \pm SD, unless otherwise stated. Descriptive statistics stratified by tuberculosis (TB) screening, infection (TB or latent tuberculosis infection (LTBI)) and exposure, and crude and adjusted p-values of the variables' effects on each of the three responses. HCW: healthcare worker; NA: not applicable. ${ }^{\#}$ : medical or intensive care. 
Regional differences in the response rates were initially detected but failed to become statistically significant in the regression models. The results can be extrapolated to the working population.

The major reason for the absence of screening was it not being offered. Post-exposure screening revealed a higher incidence of LTBI than routine screening $(\mathrm{p}=0.003)$ and treatment compliance was higher post-exposure (OR 2.9327, 95\% CI 1.7154-5.0137). The most frequently screened individuals were of intermediate age (OR 1.419, 95\% CI 1.097-1.836), worked in a hospital (OR 2.264, 95\% CI 1.833-2.798), were female (OR 1.284, 95\% CI 1.033-1.597), worked in nonsurgical services (OR 1.553, 95\% CI 1.1952.020) and had been previously exposed to TB (OR 1.858, 95\% CI 1.395-2.474). Exposure occurred most frequently in older subjects (OR 1.705, 95\% CI 1.230-2.363), those who worked in nonsurgical services (OR 1.553, 95\% CI 1.195-2.020) and hospital workers (OR 1.729, 95\% CI 1.253-2.387). Infection rates were higher in middle-aged individuals (OR 2.628, 95\% CI 1.713-4.031), hospital workers (OR 1.745, 95\% CI 1.218-2.499), workers in nonsurgical services (OR 2.398, 95\% CI 1.393-4.132) and those previously exposed to TB (OR 2.053, 95\% CI 1.062-3.967).

Concern about the side-effects of treatment was the factor cited most by individuals who refused screening, as well as those who opted not to be treated for LTBI. Similar results were reported previously [7], in that only $48.9 \%$ of 235 exposed HCWs with negative or unknown pre-exposure TST status had post-exposure TST tested. Another study reported that compliance with TST screening was very low (12.3\%), and that increased information about transmission and testing did not increase compliance [5].

One striking observation was that most unscreened professionals were not offered screening. This finding suggests that institutional measures are insufficient or incorrectly applied.

We also found that treatment compliance was low (51.8\%). Although we do not have data about whether treatment was not started or not completed, the percentage of noncompliant individuals was higher than in a previous study [7], in which 46 (93.9\%) out of 49 HCWs prescribed treatment actually started treatment but $82.6 \%$ of those failed to complete treatment. Similar results were observed in non-HCW individuals [13], in that treatment completion rates were higher after exposure to TB. We found that the percentage treated for LTBI was almost three-fold higher in HCWs diagnosed after exposure than after routine screening.

Taken together, these results indicate that physicians and nurses did not consider LTBI treatment as an important measure to avoid $\mathrm{TB}$, with the side-effects of treatment being the most frequent reason for noncompliance. This behaviour was similar to that in a previous survey [6], in which compliance was even lower, with only about $25 \%$ of physicians who were indicated for LTBI treatment completing it.

It is surprising that some professionals stated they did not receive training in $\mathrm{TB}$, suggesting that they may not regard graduate education as training or that curricula are insufficient. This factor may explain why HCWs are unaware of TB epidemiology and pathogenesis and did not recognise the consequences of latent TB. A study assessing LTBI treatment of immigrants [14] also found that providers lacked knowledge of TB, both in written tests and in practice.

We could not determine whether TB in these HCWs was mainly due to nosocomial exposure, since about $50 \%$ of affected individuals reported having TB before starting professional activity, as did one third of individuals with LTBI. This reflects the higher risk in community settings of countries with an intermediate incidence of TB [15]. Our findings indicate, however, that most affected HCWs were identified during their first years of professional activity.

The study had several limitations. The survey was electronically distributed, anonymous and self-reported; thus, the validity of the answers could not be confirmed. The survey did not differentiate among screening methods or ask about what was considered positive screening. Moreover, the survey did not differentiate individuals who did and did not adhere to treatment, nor did it evaluate treatment regimens. Although it would be interesting to follow individuals who had LTBI and find differences in their development of TB, the survey was anonymous, preventing such follow-up.

Finding the actual barriers to screening of HCW is paramount. Our study suggest that institutions should have more effective screening programmes and HCWs should receive proper training allowing them to make more informed decisions.

Wider studies throughout Europe should be developed to evaluate these issues. 

informed decisions http://ow.ly/BSitS

José Moura Meireles ${ }^{1,2}$, Rita Gaio ${ }^{3,4}$ and Raquel Duarte ${ }^{2,5-7}$

${ }^{1}$ Dept of Internal Medicine, Centro Hospitalar do Porto, Porto, Portugal. ${ }^{2}$ EPIUnit Institute of Public Health, University of Porto, Porto, Portugal. ${ }^{3}$ Mathematics Dept, Faculty of Sciences, University of Porto, Porto, Portugal. ${ }^{4}$ Centre of Mathematics, University of Porto, Portugal. ${ }^{5}$ Chest Disease Centre, Vila Nova de Gaia, Portugal. ${ }^{6}$ Dept of Pulmonology, Centro Hospitalar de Vila Nova de Gaia/Espinho EPE, Vila Nova de Gaia, Portugal. 7 Dept of Clinical Epidemiology, Predictive Medicine and Public Health, University of Porto Medical School, Porto, Portugal.

Correspondence: José Moura Meireles, Dept of Internal Medicine, Centro Hospitalar do Porto, Largo Prof. Abel Salazar, Porto, Portugal. Email, zemeireles@gmail.com

Received: July 182014 | Accepted after revision: Sept 082014 | First published online: Oct 162014

Support statement: R. Gaio was partially funded by the European Regional Development Fund through the programme COMPETE and by the Portuguese Government through the FCT - Fundação para a Ciência e a Tecnologia under the project PEst-C/MAT/UI0144/2013.

Conflict of interest: None declared.

Acknowledgements: The authors would like to thank all healthcare workers who voluntarily responded to the survey.

\section{References}

1 Menzies D, Joshi R, Pai M. Risk of tuberculosis infection and disease associated with work in health care settings. Int J Tuberc Lung Dis 2007; 11: 593-605.

2 Seidler A, Nienhaus A, Diel R. Review of epidemiological studies on the occupational risk of tuberculosis in low-incidence areas. Respiration 2005; 72: 431-446.

3 Baussano I, Nunn P, Williams B, et al. Tuberculosis among health care workers. Emerg Infect Dis 2011; 17: 488-494.

4 Bratcher DF, Stover BH, Lane NE, et al. Compliance with national recommendations for tuberculosis screening and immunization of healthcare workers in a children's hospital. Infect Control Hosp Epidemiol 2000; 21: 338-340.

5 Taubman D, Titler N, Edelstein H, et al. Providing detailed information about latent tuberculosis and compliance with the PPD test among healthcare workers in Israel: a randomized controlled study. J Epidemiol Glob Health 2013; 3: 253-260.

6 Bhanot N, Haran M, Lodha A, et al. Physicians' attitudes towards self-treatment of latent tuberculosis. Int J Tuberc Lung Dis 2012; 16: 169-171.

7 Balkhy $\mathrm{HH}$, Miller TL, Ali S, et al. Compliance with postexposure screening and treatment of latent tuberculosis infection among healthcare workers in a tertiary care hospital in Saudi Arabia. Infect Control Hosp Epidemiol 2014; 35: 176-181.

8 Torres Costa J, Silva R, Ferreira J, et al. Tuberculose ativa entre profissionais de saúde em Portugal [Active tuberculosis among health care workers in Portugal]. J Bras Pneumol 2010; 37: 636-645.

9 Diel R, Loddenkemper R, Zellweger J-P, et al. Old ideas to innovate tuberculosis control: preventive treatment to achieve elimination. Eur Respir J 2013; 42: 785-801.

10 D'Ambrosio L, Dara M, Tadolini M, et al. Tuberculosis elimination: theory and practice in Europe. Eur Respir J 2014; 43: 1410-1420.

11 R Development CoreTeam. R: A Language and Environment for Statistical Computing. Vienna, R Foundation for Statistical Computing, 2013.

12 Direcção de Serviços de Epidemiologiae Estatísticas de Saúde, Divisão de Estatísticas de Saúde. Elementos estatísticos: informação geral - saúde 2008 [Statistical data: general information - health 2008]. Lisbon, Direcção-Geral da Saúde, 2010.

13 Li J, Munsiff SS, Tarantino T, et al. Adherence to treatment of latent tuberculosis infection in a clinical population in New York City. Int J Infect Dis 2010; 14: e292-e297.

14 Hill L, Blumberg E, Sipan C, et al. Multi-level barriers to LTBI treatment: a research note. J Immigr Minor Health 2010; 12: 544-550.

15 Hung W-T, Lee SS-J, Sy C-L, et al. Prevalence of latent tuberculosis infection in BCG-vaccinated healthcare workers by using an interferon-gamma release assay and the tuberculin skin test in an intermediate tuberculosis burden country. J Microbiol Immunol Infect 2013: pii: S1684-1182(13)00124-2. 